\title{
Switch from tablet levothyroxine to oral solution resolved reduced absorption by intestinal parasitosis
}

\author{
AnnaTortora, DomenicoLa Sala and MarioVitale \\ Department of Medicine, Surgery and Dentistry, University of Salerno, Baronissi, Salerno, Italy
}

Correspondence should be addressed to M Vitale

Email

mavitale@unisa.it

\section{Summary}

Reduced intestinal absorption of levothyroxine (LT4) is the most common cause of failure to achieve an adequate therapeutic target in hypothyroid patients under replacement therapy. We present the case of a 63-year-old woman with autoimmune hypothyroidism previously well-replaced with tablet LT4 who became unexpectedly no more euthyroid. At presentation, the patient reported the onset of acute gastrointestinal symptoms characterized by nausea, loss of appetite, flatulence, abdominal cramps and diarrhea, associated with increase of thyrotropin levels (TSH: $11 \mathrm{mIU} / \mathrm{mL}$ ). Suspecting a malabsorption disease, a thyroxine solid-to-liquid formulation switch, at the same daily dose, was adopted to reach an optimal therapeutic target despite the gastrointestinal symptoms persistence. Oral LT4 solution normalized thyroid hormones. Further investigations diagnosed giardiasis, and antibiotic therapy was prescribed. This case report is compatible with a malabsorption syndrome caused by an intestinal parasite (Giardia lamblia). The reduced absorption of levothyroxine was resolved by LT4 oral solution.

\section{Learning points:}

- The failure to adequately control hypothyroidism with oral levothyroxine is a common clinical problem.

- Before increasing levothyroxine dose in a patient with hypothyroidism previously well-controlled with LT4 tablets but no more in appropriate therapeutic target, we suggest to investigate non adhesion to LT4 therapy, drug or food interference with levothyroxine absorption, intestinal infection, inflammatory intestinal disease, celiac disease, lactose intolerance, short bowel syndrome after intestinal or bariatric surgery, hepatic cirrhosis and congestive heart failure.

- LT4 oral solution has a better absorptive profile than the tablet. In hypothyroid patients affected by malabsorption syndrome, switch of replacement therapy from tablet to liquid LT4 should be tested before increasing the dose of LT4.

\section{Background}

Oral levothyroxine (LT4), used to treat hypothyroidism, is absorbed through the intestinal mucosa at the level of the duodenum, jejunum and ileum. Only a fraction of the ingested dose is absorbed, depending on a number of factors including the age of the patient, dietary habits, malabsorption and interference of other drugs.

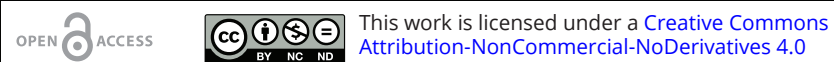
International License.
}

To be absorbed, LT4 ingested as tablet formulation requires dissolution in acid environment. Subjects with achlorhydria or reduced gastric acidity or patients treated with proton-pump inhibitors have a reduced LT4 absorption due to reduced gastric LT4 dissolution. Recently developed liquid LT4 formulation has the advantage that does not require stomach acidity to be dissolved and is 
ready to be absorbed through the intestinal mucosa. In this formulation, LT4 is dissolved in an alcoholic solution, its absorption is faster and independent by gastric acidity. Malabsorption of LT4 has been described in patients with gastric or intestinal disorders including infectious diseases by Giardia lamblia. We describe a case of reduced absorption of tablet LT4 in the course of intestinal giardiasis that was corrected by LT4 oral solution.

\section{Case presentation}

A 63-year-old woman affected by autoimmune chronic lymphocytic thyroiditis and hypothyroidism was followed up at our outpatient clinic by several years. The annual monitoring demonstrated a good pharmacological control by tablet LT4 with thyroid hormones and thyroid-stimulating hormone (TSH) serum levels within the normal range (Table 1). She reported a positive family history for autoimmune thyroid disease, one term pregnancy and menopause at the age of 51 years. The patient was not a smoker and denied specific food or drug allergies, previous traumas, surgery or cancer. Thyroid gland ultrasound examination revealed a slightly enlarged gland with heterogeneous pattern diffusely hypoechoic with multiple fine fibrous branches in the absence of clear thyroid nodules. Hypothyroidism was treated by daily tablet LT4 administered $60 \mathrm{~min}$ before breakfast. After many years of euthyroidism, the patient exhibited a slight increase of TSH over the normal limit $(5.5 \mathrm{mIU} / \mathrm{mL})$ with free thyroid hormones within the reference range (Table 2). The patient stated that neither the replacement therapy, nor her diet and habits were changed. Weight was stable and neck ultrasound examination showed no significant changes of thyroid echostructure with respect to the past. For this reason we confirmed the LT4 replacement dosage $(1.2 \mu \mathrm{g} / \mathrm{kg} / \mathrm{day})$ and invited the patient to a short interval follow-up of about 2 months. Two months later, the woman reported to us the onset of gastrointestinal symptoms, initiated 3 weeks before, including nausea, weight loss, flatulence, abdominal cramps, fatigue and watery diarrhea without mucus or blood. She claimed to have not travelled during the last year, and neither changed her food or pharmacological habits nor missed her LT4 daily dose. The patient clinical evaluation showed a weight of $60 \mathrm{~kg}$ (two $\mathrm{kg}$ less than the previous control), BMI $22.5 \mathrm{~kg} /$ $\mathrm{m}^{2}$, blood pressure $125 / 75 \mathrm{mmHg}$ and heart rate of 74 beats per minute. Complete blood count, glycemia, creatinine, transaminases and stool analysis (this one on a single sample) were normal, while thyroid function tests showed inadequate therapeutic compensation with TSH values higher than the reference limits $(11 \mathrm{mIU} / \mathrm{mL})$. Suspecting a malabsorption condition, we replaced LT4 tablets with its liquid solution while keeping the same dosage per $\mathrm{kg}$ /body weight (Table 2). After 2 months, the woman reported the persistence of intestinal symptoms for which we recommended blood examinations, stool analysis and abdominal ultrasound, but the thyroid hormonal exams she exhibited were considerably improved and actually within normal limits (TSH: $3.1 \mathrm{mIU} / \mathrm{mL}$ ). For this reason, we confirmed the same LT4 replacement therapy both for the liquid formulation and for the dosage (Table 2). On the next visit, after about 3 months, our patient exhibited stool analysis (on three samples) positive for Giardia lamblia, parasite contracted from her domestic dog which was the reservoir of this infection. Her blood values were normal except for the serum protein electrophoresis which showed a slight hypoalbuminemia and for the thyroid hormonal exams not yet in adequately therapeutic target (TSH: $4.1 \mathrm{mIU} /$ $\mathrm{mL}$ ). Therefore, we slightly increased LT4 dose of oral solution and prescribed specific antibiotic (metronidazole $250 \mathrm{mg}$ three times a day for seven days) for the Giardiasis. During the following clinical control after 2 months, the woman reported complete resolution of gastrointestinal symptoms confirmed by a negative stool analysis (on three samples) for parasitological infection, while TSH was $0.91 \mathrm{mIU} / \mathrm{mL}$. Considering the parasite eradication, the resolution of malabsorption syndrome and that TSH was lower than the age-appropriate therapeutic target, we reduced the LT4 dosage maintaining however its liquid formulation that was more effective than tablet T4 during the acute parasitic infestation; on the next visit after about 6 months, the patient reported psycho-physical

Table 1 TSH, thyroid hormone status and replacement therapy with levothyroxine.

\begin{tabular}{lcc}
\hline Date & Weight $(\mathrm{kg})$ \\
${$\cline { 1 - 1 } 2015$} }$ & 62 \\
June 2016 & 62 \\
Normal range & \\
\hline
\end{tabular}

\begin{tabular}{ccc}
\hline $\mathbf{T S H}(\mathrm{mlU} / \mathrm{mL})$ & & $\mathbf{F T 3}(\mathrm{pg} / \mathrm{mL})$ \\
\cline { 1 - 1 } 2.7 & & 2.6 \\
2.8 & & 3.8 \\
$0.35-5.5$ & & $2.2-4.5$ \\
\hline
\end{tabular}

\begin{tabular}{c}
\hline FT4 $(\mathrm{ng} / \mathrm{mL})$ \\
\hline 1.1 \\
1.4 \\
$0.9-1.8$ \\
\hline
\end{tabular}

\begin{tabular}{c}
\hline $\mathbf{A b T g}(\mathrm{IU} / \mathrm{mL})$ \\
\hline 415 \\
$0-115$ \\
\hline
\end{tabular}

AbTPO $(\mathrm{IU} / \mathrm{mL})$
119
$0-34$
$\frac{\mathbf{L T 4}(\mu \mathrm{g} / \mathrm{kg} / \mathrm{day})}{1.2}$ 1.2

AbTg, anti-thyroglobulin antibodies; AbTPO, anti thyroperoxidase antibodies; TSH, thyroid-stimulating hormone. 
Table 2 Thyroid hormonal exams and replacement therapy with tablet and oral solution levothyroxine.

\begin{tabular}{lcc}
\hline Timeline/daily therapy with L-T4 & & Weight $(\mathrm{kg})$ \\
\hline Normal range & & 62 \\
Sept 2017* & 60 \\
60 days after tablets $(1.2 \mu \mathrm{g} / \mathrm{kg})$ & 60 \\
60 days after oral solution $(1.2 \mu \mathrm{g} / \mathrm{kg})$ & & 59 \\
90 days after oral solution $(1.4 \mu \mathrm{g} / \mathrm{kg})$ & & 59 \\
Start metronidazole & & 60 \\
$\quad 60$ days after oral solution $(1.2 \mu \mathrm{g} / \mathrm{kg})$ & \\
6 months after oral solution $(1.2 \mu \mathrm{g} / \mathrm{kg})$ &
\end{tabular}

*Baseline.

well-being and exhibited thyroid hormonal exams in therapeutic target (TSH: $1.2 \mathrm{mIU} / \mathrm{mL}$ ) (Table 2).

\section{Investigation}

The coexistence of gastrointestinal symptoms and increased serum TSH induced us to suspect an acute condition of malabsorption. Routine exams and stool analysis allowed us to identify Giardia lamblia as responsible of intestinal infection.

\section{Treatment}

Our patient had been undergoing replacement therapy with LT4 tablets at a dose of $1.2 \mu \mathrm{g} / \mathrm{kg} /$ day for many years. After the onset of gastrointestinal symptoms, we switched to oral solution of levothyroxine keeping the same dose per $\mathrm{kg}$ of body weight $(1.2 \mu \mathrm{g} / \mathrm{kg} /$ day). Following the diagnoses of Giardiasis we prescribed specific eradication treatment: metronidazole $250 \mathrm{mg}$ three times/day for 7 days. At the same time, we increased LT4 dose to $1.4 \mu \mathrm{g} / \mathrm{kg} /$ day maintaining the liquid formulation to achieve a better therapeutic compensation. After about 60 days, because of the TSH levels at the lower limit of normal, we reduced LT4 to $1.2 \mu \mathrm{g} / \mathrm{kg} /$ day with subsequent achievement of optimal therapeutic target maintained during the time.

\section{Outcome and follow-up}

The switch from LT4 tablets to LT4 oral solution normalized serum thyroid hormones and TSH, despite the malabsorption syndrome induced by Giardia lamblia infection. Optimal therapeutic target was maintained during the time.

\section{Discussion}

Levothyroxine is one of the most prescribed drugs worldwide. The classic formulation of LT4 is tablet, containing a sodium salt of LT4 and several excipients.
An acid gastric $\mathrm{pH}$ is required to permit dissolution and its arrival in the intestine, where LT4 is absorbed (1). LT4 permeation through the intestinal epithelium mainly occurs in the duodenum and the jejunum, mostly within the first $3 \mathrm{~h}$ after ingestion, while the fastest part of the intestinal absorption happens within the first $2 \mathrm{~h}(1,2)$. Approximately $70 \%$ of the LT 4 contained in the tablet is absorbed and the best timing of LT4 ingestion is in the morning, at least $1 \mathrm{~h}$ before breakfast (3). LT4 intestinal absorption can be impaired by some beverages (coffee, grapefruit), by different gastrointestinal diseases or surgical interventions (celiac disease, bariatric surgery) and by drugs that physically sequester the hormone (aluminum hydroxide, ferrous sulfate, cholestyramine, calcium carbonate) or increase the gastric $\mathrm{pH}$ (proton-pump inhibitors) (4). Recently, new LT4 formulations, the soft gel capsule and the oral solution, have become available (5, 6, 7). In the oral solution, LT4 is solubilized in $95 \%$ ethanol and $86 \%$ glycerol and needs no dissolution; therefore, it is absorbed faster than tablet and in one-sixth of patients, the daily dose has to be decreased (personal observation). Oral liquid levothyroxine is demonstrated to be useful to overcome the interference exerted by the concurrent ingestion of interfering drugs (8). The failure to adequately control hypothyroidism with oral levothyroxine is a common clinical problem; on this point, non-adhesion to prescription is reported as the most common cause, but other conditions can be responsible of LT4 reduced absorption. Withdrawal of the interfering drugs and identification of conditions of malabsorption should be investigated before increasing LT4 dosage in order to avoid the risk of iatrogenic hyperthyroidism. Routinely ordered tests include stool analysis (for intestinal infections), antigliadin antibodies (for celiac disease), inflammation markers (for inflammatory intestinal disease), lactose tolerance test, urea breath test for Helicobacter pylori infection (9) and, occasionally, anti-T4 antibodies (10). Short bowel syndrome after intestinal or bariatric surgery, hepatic cirrhosis and congestive heart failure can also impair LT4 intestinal 
absorption (11). Giardiasis, a common intestinal infection, has been rarely reported to impair the absorption of orally ingested LT4 $(12,13)$. Giardiasis is probably sub-diagnosed, but its prevalence is about $20 \%$ in developing countries because of their poor sanitary conditions, while it is rather variable, from $3 \%$ to $7 \%$, in industrialized countries. Most patients infected by Giardia lamblia remain asymptomatic for variable time before presenting diarrhea, flatulence and abdominal pain. A known complication of chronic intestinal giardiasis is malabsorption of drugs and nutrients, leading to anemia, weight loss and growth retardation. The presence of trophozoites activates intestinal T lymphocytes, which induce disruption of epithelial tight junctions and enterocitic apoptosis. This inflammatory epithelial damage results in loss of epithelial barrier and increased intestinal permeability leading to diffuse shortening of microvilli, maldigestion, malabsorption and diarrhea (14). Giardia lamblia infection should be suspected in the presence of these symptoms. In this clinical case, the switch from tablet LT4 to its oral solution reverted the decreased levothyroxine absorption. One possible explanation of the reduced absorption of the solid LT4 formulation is that the intestinal inflammatory state extended to the stomach, reducing its acidity, conditioning its emptying and finally reducing the dissolution of LT4.

In conclusion, Giardiasis can reduce tablet LT4 absorption in hypothyroid patients. Subjects with previously well-controlled hypothyroidism with LT4 tablet, with typical symptoms of giardiasis (weight loss, diarrhea, abdominal pain, anemia) or with personal or family history of giardiasis must be suspected and investigated. In hypothyroid patients affected by giardiasis and levothyroxine malabsorption, switch of replacement therapy from tablet to oral solution LT4 should be tested before increasing the dose of LT4.

\section{Declaration of interest}

The authors declare that there is no conflict of interest that could be perceived as prejudicing the impartiality of the research reported.

\section{Funding}

This research did not receive any specific grant from any funding agency in the public, commercial or not-for-profit sector.

\section{Patient consent}

A written informed consent has been obtained from the patient for publication of the submitted article.

\section{Author contribution statement}

All authors were in charge of patient's management. A T wrote the initial draft of the article and contributed substantially to writing the case report. $\mathrm{M} \vee$ prepared the final manuscript.

\section{References}

1 Hays MT. Localization of human thyroxine absorption. Thyroid 1991 1 241-248. (https://doi.org/10.1089/thy.1991.1.241)

2 Colucci P, Yue CS, Ducharme M \& Benvenga S. A review of the pharmacokinetics of levothyroxine for the treatment of hypothyroidism. European Endocrinology 20139 40-47. (https://doi. org/10.17925/EE.2013.09.01.40)

3 Bach-Huynh TG, Nayak B, Loh J, Soldin S \& Jonklaas J. Timing of levothyroxine administration affects serum thyrotropin concentration. Journal of Clinical Endocrinology and Metabolism 2009 94 3905-3912. (https://doi.org/10.1210/jc.2009-0860)

4 Virili C, Antonelli A, Santaguida MG, Benvenga S \& Centanni M. Gastrointestinal malabsorption of thyroxine. Endocrine Reviews 2019 40 118-136. (https://doi.org/10.1210/er.2018-00168)

5 Benvenga S, Bartolone L, Pappalardo MA, Russo A, Lapa D, Giorgianni G, Saraceno G \& Trimarchi F. Altered intestinal absorption of L-thyroxine caused by coffee. Thyroid 200818 293-301. (https://doi.org/10.1089/thy.2007.0222)

6 Virili C, Trimboli P, Romanelli F \& Centanni M. Liquid and softgel levothyroxine use in clinical practice: state of the art. Endocrine 2016 54 3-14. (https://doi.org/10.1007/s12020-016-1035-1)

7 Vita R, Fallahi P, Antonelli A \& Benvenga S. The administration of L-thyroxine as soft gel capsule or liquid solution. Expert Opinion on Drug Delivery 201411 1103-1111. (https://doi.org/10.1517/1742524 7.2014.918101)

8 Vita R, Di Bari F \& Benvenga S. Oral liquid levothyroxine solves the problem of tablet levothyroxine malabsorption due to concomitant intake of multiple drugs. Expert Opinion on Drug Delivery 201714 467-472. (https://doi.org/10.1080/17425247.2017.1290604)

9 Lahner E, Virili C, Santaguida MG, Annibale B \& Centanni M. Helicobacter pylori infection and drugs malabsorption. World Journal of Gastroenterology 201420 10331-10337. (https://doi.org/10.3748/ wjg.v20.i30.10331)

10 Suzuki Y, Takeshita E, Kano S, Hirata S \& Sato S. Impaired intestinal absorption of thyroid hormone in a case of Hashimoto's disease with anti-T3 and anti-T4. Nippon Naibunpi Gakkai Zasshi 198258 1487-1497.

11 Padwal R, Brocks D \& Sharma AM. A systematic review of drug absorption following bariatric surgery and its theoretical implications. Obesity Reviews 201011 41-50. (https://doi. org/10.1111/j.1467-789X.2009.00614.x)

12 Radaeli RdeF \& Diehl LA. Increased levothyroxine requirement in a woman with previously well-controlled hypothyroidism and intestinal giardiasis. Arquivos Brasileiros de Endocrinologia e Metabologia 201155 81-84. (https://doi.org/10.1590/S000427302011000100012)

13 Seppel T, Rose F \& Schlaghecke R. Chronic intestinal giardiasis with isolated levothyroxine malabsorption as reason for severe hypothyroidism implications for localization of thyroid hormone absorption in the gut. Experimental and Clinical Endocrinology and Diabetes 1996104 180-182. (https://doi.org/10.1055/s-0029-1211442)

14 Buret AG. Pathophysiology of enteric infections with Giardia duodenalis. Parasite 200815 261-265. (https://doi.org/10.1051/ parasite/2008153261)

Received in final form 25 February 2019

Accepted 4 March 2019 